Isabel Aninat y Slaven Razmilic (coordinadores), Un Estado para la ciudadanía. Informe de la Comisión de Modernización del Estado (Santiago: Centro de Estudios Públicos, 2017).

Isabel Aninat y Slaven Razmilic (editores), Un Estado para la ciudadanía. Estudios para su modernización (Santiago: Centro de Estudios Públicos, 2018).

RESEÑA

\title{
MODERNIZACIÓN DEL ESTADO: LOS APORTES DE LA FLEXIBILIDAD EN EL MANEJO PRESUPUESTARIO, LA EVALUACIÓN Y EL PRESUPUESTO POR RESULTADO
}

\author{
Heidi Berner Herrera \\ Facultad de Economía y Negocios \\ Universidad de Chile
}

\section{INTRODUCCIÓN}

a presente reseña tiene por objetivo analizar de manera detallada las propuestas elaboradas por la Comisión de Modernización del Estado que fue convocada por el Centro de Estudios Públicos (CEP) entre julio de 2016 y octubre de 2017. En particular, este análisis se focalizará en aquellas propuestas que dicen relación con el perfeccionamiento de la gestión financiera, procesos de planificación estratégica y rendición de cuentas, y su complemento con la evaluación, control y transparencia del uso de los recursos públicos y sus resultados.

Heidi Berner Herrera. Economista de la Universidad de Chile y magíster en administración pública de la Kennedy School of Government, Harvard University. Jefa de la División de Control de Gestión de la Dirección de Presupuestos del Ministerio de Hacienda en 2006-2010. Subsecretaria de Evaluación Social del Ministerio de Desarrollo Social en 2014-2018. Profesora en el magíster en políticas públicas de la Facultad de Economía y Negocios de la Universidad de Chile. Email: heidi.berner. herrera@gmail.com. 
Para ello, se tomará en cuenta, en lo que corresponda, los artículos de Gerhard Steger ("Elaboración de presupuesto por resultados: lecciones de la reforma presupuestaria austriaca") y de Hermann von Gersdorff ("El proceso presupuestario en Chile: opciones de reforma"), estudios que se escribieron para apoyar el trabajo de la Comisión convocada por el CEP. Sumo, además, "Budgeting in Chile", elaborado por la OECD en 2017. ${ }^{1}$

Es importante señalar que el análisis se realizará considerando la experiencia de la autora como coordinadora de evaluaciones de impacto en la Dirección de Presupuestos del Gobierno de Chile (Dipres); como jefa, más tarde, de la división de control de gestión de la misma Dipres, y, luego, como subsecretaria de Evaluación Social del Ministerio de Desarrollo Social. Ello le permite tener la mirada de quien realiza el monitoreo y evaluación de los programas y proyectos de inversión pública, así como de una jefa de servicio que debe ejecutar un presupuesto y cumplir con las metas propuestas.

Se considera que, tal como lo plantea la Comisión convocada por CEP - en adelante la Comisión-, la modernización del Estado debe ser una tarea permanente, que involucre el perfeccionamiento continuo de la acción de los distintos ministerios y servicios, con miras a responder las necesidades y expectativas de los ciudadanos, con foco en la prestación de servicios orientada a mejorar sus niveles de satisfacción.

Todas las propuestas de modernización que se plantean en el ámbito de la gestión financiera, procesos de planificación estratégica y rendición de cuentas y evaluación, control y transparencia van justamente en la línea de un Estado en el que el perfeccionamiento continuo es una tarea primordial, y más que una reforma estructural del mismo.

En materia de gestión financiera se plantean propuestas de modernización en cuatro ámbitos: i) crear incentivos al ahorro en la gestión presupuestaria; ii) generar más espacios de flexibilidad en la ejecución del presupuesto; iii) reducir los trámites para agilizar la gestión y la ejecución presupuestaria, y iv) integrar temáticamente el proceso de formulación, discusión y supervisión del presupuesto.

En materia de gestión organizacional, se entregan cinco propuestas: i) introducir la planificación estratégica con énfasis en gestión de

${ }^{1}$ Camila Vammalle y Ana María Ruiz Rivadeneira, "Budgeting in Chile", OECD Journal on Budgeting 2016, n. 3 (2017). 
procesos y rendición de cuentas; ii) institucionalizar el Consejo de $\mathrm{Au}$ ditoría Interna General de Gobierno, fortaleciendo su rol colaborador y preventivo; iii) continuar con las mejoras de ChileCompra; iv) reformar la institucionalidad y competencias del Tribunal de Compras Públicas, y v) implementar, en la Secretaría General de la Presidencia (Segpres), una plataforma de servicios compartidos para una mejor regulación.

En particular, para efectos de esta reseña, se analizará la propuesta de la introducción de la planificación estratégica con énfasis en gestión de procesos y rendición de cuentas, ya que ésta se relaciona íntegramente con las propuestas en el ámbito de gestión financiera y en el ámbito de evaluación, control y transparencia.

Las propuestas en este último ámbito de evaluación, control y transparencia son seis: i) institucionalizar la Comisión de Productividad y extender sus facultades, estableciendo la Agencia de Productividad y Evaluación; ii) promover modificaciones a la forma de control que ejerce la Contraloría General de la República (CGR); iii) rediseñar el sistema de control de gestión pública; iv) aumentar la transparencia y mejorar la calidad de la información de evaluación de desempeño; v) velar por la usabilidad de los datos que se transparentan, y vi) establecer plataformas comunes para la medición de satisfacción de los ciudadanos.

Considerando su relación con la gestión financiera y la rendición de cuentas, en esta reseña se abordarán con mayor profundidad las propuestas en materia de la creación de la Agencia de Productividad y Evaluación, el perfeccionamiento del sistema de control de gestión pública y de la calidad de la información de desempeño que se entrega.

Para analizar las propuestas antes mencionadas, se seguirá un orden distinto al planteado en el Informe de la Comisión, pues el punto de partida para generar una gestión financiera con incentivos al ahorro de recursos y mayor flexibilidad en el manejo presupuestario debe ser el contar con una planificación estratégica, que defina los principales productos — bienes y servicios - que se entregan y que estos productos puedan ser fácilmente vinculados con los recursos asignados a través de la Ley de Presupuestos de cada año, y evaluados en materia de eficacia, eficiencia y calidad (oportunidad y satisfacción en la entrega), de manera de hacer seguimiento al cumplimiento de las metas comprometidas, además de sus resultados de mediano y largo plazo (impacto). 


\section{INTRODUCIR LA PLANIFICACIÓN ESTRATÉGICA CON ÉNFASIS EN GESTIÓN DE PROCESOS Y RENDICIÓN DE CUENTAS}

El Informe de la Comisión plantea que, "en la actualidad, la mayoría de los servicios no tiene marcos que establezcan de manera clara, pública e integrada los objetivos que persiguen, los recursos que disponen para alcanzarlos y la forma en que estos medios se articulan en pos de ese objetivo"; 2 sin embargo, es preciso señalar que el proceso de formulación del presupuesto de cada año obliga a las instituciones públicas (subsecretarías y servicios) al establecimiento de sus definiciones estratégicas (formulario A-1), las que incluyen la misión institucional, junto a la identificación de sus objetivos estratégicos, productos estratégicos y los clientes, beneficiarios o usuarios a los cuales están destinados dichos productos. Esta obligación se establece a partir del año 2000, junto con el rediseño del Programa de Mejoramiento de la Gestión (PMG), ${ }^{3}$ que permitió comenzar con la implementación en las instituciones públicas de sistemas de gestión, dentro de los que se consideraba como fundamental el contar con un sistema de Planificación y Control de Gestión. Adicionalmente, desde el mismo año, las instituciones públicas comprometen anualmente indicadores y metas como parte del proceso de formulación presupuestaria (formulario H). Dichos indicadores buscan hacer seguimiento al cumplimiento de los productos estratégicos (bienes o servicios que se entregan), y ser parte del sistema de Planificación y Control de Gestión.

La revisión de las definiciones estratégicas se realiza anualmente, en búsqueda de su perfeccionamiento, considerando las prioridades gubernamentales trabajadas con el Ministerio Secretaría General de la Presidencia y las prioridades presupuestarias establecidas en el proyecto de Ley de Presupuestos, de modo que reflejen adecuadamente la gestión relevante de la institución y los recursos asignados.

2 Isabel Aninat y Slaven Razmilic (coordinadores), Un Estado para la ciudadanía. Informe de la Comisión de Modernización del Estado (Santiago: Centro de Estudios Públicos, 2017), 106. En adelante, las citas a este documento se harán tan sólo con el número de página entre paréntesis.

${ }^{3}$ Los Programas de Mejoramiento de la Gestión (PMG) en los servicios públicos tienen su origen en la Ley 19.553, de 1998, modificada por las leyes 19.618, 19.882 y 20.212 , que asocian el cumplimiento de objetivos de gestión a un incentivo de carácter monetario para los funcionarios. 
Estas definiciones estratégicas son la base del sistema de Planificación y Control de Gestión, que, según ya se señaló, era parte de los sistemas de gestión del PMG. Para asegurar que el sistema de planificación y control de gestión de cada institución permitiera, además de medir los aspectos esenciales del desempeño a través de indicadores, la identificación de los procesos involucrados en la generación de cada uno de los productos estratégicos - como lo recomienda el Informe de la Comisión-, en 2005 se inició el proceso de certificación, bajo la norma ISO 9001, de los sistemas de gestión del PMG, utilizando para ello toda la estructura y organización de la ISO internacional, apoyando a los servicios públicos con instituciones especialistas en la preparación de un proceso de certificación, y con instituciones acreditadas para la certificación ISO. Posteriormente, en 2009 se incorporó al PMG la certificación bajo la norma ISO 9001 de los procesos relacionados con la provisión de bienes y servicios relevantes para el desempeño de la institución, considerando la perspectiva territorial y de género, como también los sistemas de gestión que eran parte del PMG. ${ }^{4}$ En 2009, 42 servicios de los 198 que eran parte del PMG - y por tanto contaban con sistemas de planificación y control de gestión- se encontraban en la etapa de certificación de los procesos de producción de los bienes y servicios que entregaban.

Actualmente, el PMG no se basa en sistemas de gestión ni en la certificación bajo la norma ISO de los procesos que permiten la generación de cada uno de los productos estratégicos, aun cuando mantiene el establecimiento de indicadores y metas en el formulario $\mathrm{H}$ para vincular la evaluación de su cumplimiento con el incentivo asociado al PMG. Ésta puede ser una de las razones que llevan a la Comisión a plantear que no siempre las definiciones estratégicas contienen información relevante, y que ellas no se relacionan necesariamente con la gestión de los servicios, las acciones realizadas y los recursos asignados (106). Sin embargo, se debe reconocer que existe heterogeneidad en la calidad de las definiciones estratégicas elaboradas por los servicios, tanto respecto de la vinculación de los productos estratégicos con los programas pre-

${ }^{4}$ En Alberto Arenas y Heidi Berner, Presupuesto por resultados y la consolidación del Sistema de Evaluación y Control de Gestión del gobierno central (Santiago: Dirección de Presupuestos, Ministerio de Hacienda de Chile, 2010), 40, 52 y 53. 
supuestarios y los recursos involucrados en su provisión, como en la definición de los indicadores de desempeño que permiten hacer seguimiento al logro de los productos definidos.

Por tanto, se comparte la propuesta de la Comisión que señala que los servicios deben realizar procesos de planificación estratégica, identificando los objetivos que permiten la generación de sus productos, los recursos involucrados y los resultados perseguidos (107). Sin embargo, su implementación debe considerar lo avanzado a partir de la elaboración de las definiciones estratégicas e indicadores que acompañan la formulación presupuestaria, además de exigir que estas definiciones estratégicas sean parte del sistema de planificación y control de gestión de cada institución. Para que esta exigencia sea efectiva, se debe vincular dicha planificación con el resto del sistema de control de gestión pública, y, en particular, a los mecanismos existentes para la evaluación del desempeño de los funcionarios, como PMG y los convenios de desempeño, no sólo de los altos directivos públicos, sino también los convenios de desempeño colectivo que involucran a todos los funcionarios. ${ }^{5}$ Esto es especialmente relevante cuando se considera que la Ley 19.553, en su artículo 7 letra d), establece que las metas y sus indicadores, incorporados en el convenio de desempeño colectivo, deben estar vinculados con las definiciones de misión institucional, objetivos estratégicos y productos relevantes de cada ministerio o servicio, validadas en el sistema de planificación y control de gestión del PMG. Ello está en línea con la sugerencia de la Comisión respecto de que los convenios de desempeño colectivo se transforman en instrumentos útiles para la gestión.

\section{REDISEÑAR EL SISTEMA DE CONTROL DE GESTIÓN}

El Informe de la Comisión señala que "existe poca congruencia entre los productos estratégicos que persiguen los servicios y los programas presupuestarios que reconoce la Ley de Presupuestos" (127), y que esto se debería a la falta de coordinación en la formulación del presupuesto entre los equipos financieros y los de control de gestión, tanto

${ }^{5}$ La Ley 19.882, de 2003, que modificó el artículo $7^{\circ}$ de la Ley 19.553, señala que los convenios de desempeño colectivo son un mecanismo de incentivo al cumplimiento de metas, con el que, a través de un convenio entre el jefe del servicio y el ministro del ramo, se definen metas a partir de indicadores de desempeño. 
al interior de la Dipres como en los propios ministerios y servicios. Esa situación es en parte realidad, sin embargo su causa no es tan clara.

Desde el año 2010 se reconoce que un aspecto a avanzar para obtener una mayor calidad y pertinencia de los indicadores de desempeño y metas que acompañan la formulación del presupuesto es establecer una relación más clara entre los productos estratégicos, los indicadores y los programas presupuestarios; es decir, fijar con mayor claridad la relación entre asignaciones financieras y niveles de producción. ${ }^{6}$ Por tanto, otra causa de la inadecuada vinculación de los productos estratégicos con los programas presupuestarios es la diferencia en la definición de ambos conceptos. Por una parte, en el Decreto 854, del 29 de septiembre de 2004, se establece que el programa presupuestario corresponde a la división presupuestaria de los capítulos (servicios públicos), en relación con las funciones u objetivos específicos identificados dentro de los presupuestos de los organismos públicos, lo que permite distintas formas de agrupación del gasto correspondiente a un programa presupuestario. ${ }^{7}$ Por otra, la definición de producto estratégico establecida por la Dipres señala que corresponde a los bienes y servicios que la institución ofrece como respuestas a las necesidades de sus clientes, beneficiarios o usuarios. Los productos estratégicos se generan bajo la responsabilidad de la institución, ya sea por producción directa o por subcontratación. ${ }^{8}$

Sin embargo, pese a estas diferencias conceptuales, la Dipres ha venido trabajando con los ministerios y servicios en la misma línea de la recomendación señalada por la Comisión (128), avanzando gradualmente en que los programas presupuestarios sean consistentes con los productos estratégicos o viceversa, de modo de generar indicadores asociados a estos productos, con el fin de permitir un adecuado seguimiento, no sólo desde un punto de vista financiero (ejecución presu-

${ }^{6}$ En Arenas y Berner, Presupuesto por resultados, 83.

${ }^{7}$ La Ley de Presupuestos se estructura en "partidas" (en las que cada una corresponde a un ministerio o a una institución del Estado); que a su vez se dividen en "capítulos" (típicamente subsecretarías, servicios, direcciones, fondos o superintendencias); que a su vez se dividen en "programas presupuestarios", al interior de los cuales cada gasto se clasifica en "subtítulos", cada uno con sus respectivos "ítems". Los subtítulos (gasto en personal, transferencias, inversión, entre otros) son comunes a todos los programas presupuestarios, lo que permite hacer comparaciones entre distintos programas, capítulos y partidas. (N. del E.)

${ }^{8}$ Ver http://www.dipres.gob.cl/598/w3-propertyvalue-15219.html/. 
puestaria), sino también desde el punto de vista del cumplimiento de los resultados asociados a los productos (cobertura, resultados intermedios y finales, según corresponda).

Se debería esperar que este avance se reflejara especialmente en los presupuestos de los ministerios que involucran un mayor porcentaje del gasto. Al analizar el Ministerio de Educación, que representa el 23 por ciento del presupuesto en 2017, y, en particular, la Subsecretaría de Educación, con el 77 por ciento del presupuesto del Ministerio, es posible apreciar que este capítulo se divide en 12 programas presupuestarios en el presupuesto 2017, en algunos de los cuales el gasto en la entrega del servicio está en un programa presupuestario distinto de sus costos de operación. Ése es el caso, por ejemplo, del programa "subvenciones de los establecimientos educacionales" (que agrupa las múltiples subvenciones que puede recibir su establecimiento, de acuerdo a sus características y la de sus alumnos) ${ }^{9}$ y del programa "gestión de subvenciones de establecimientos educacionales". ${ }^{10}$ Lo mismo ocurre en el caso de la educación superior, en la que existen tres programas presupuestarios relacionados con los bienes y servicios que se entregan a las instituciones de educación superior o sus estudiantes: Fortalecimiento de la Educación Pública, ${ }^{11}$ Educación Superior ${ }^{12}-$ que contiene el financiamiento de gratuidad y becas entre otras materias- y Gasto de Operación de Educación Superior. ${ }^{13}$ Se considera un avance el diferenciar, en distintos programas presupuestarios, el gasto en bienes y servicios del gasto operacional, pues permite realizar un seguimiento de la eficiencia de la inversión, especialmente en materia de gastos administrativos como porcentaje del total realizado en la provisión de los bienes y servicios, tal como lo sugiere en su informe la OECD.

Sin embargo, al analizar las definiciones estratégicas asociadas a la Subsecretaría de Educación en $2017^{14}$ se señala la existencia de cinco productos estratégicos: i) políticas y normas educativas, ii) gestión y apoyo educativo, iii) sistema de financiamiento, iv) servicios asisten-

\footnotetext{
${ }^{9}$ Ley de Presupuestos 2017, partida 09, capítulo 01, programa 20.

${ }^{10}$ Ley de Presupuestos 2017, partida 09, capítulo 01, programa 21.

${ }^{11}$ Ley de Presupuestos 2017, partida 09, capítulo 01, programa 29.

${ }^{12}$ Ley de Presupuestos 2017, partida 09, capítulo 01, programa 30.

${ }^{13}$ Ley de Presupuestos 2017, partida 09, capítulo 01, programa 31.

${ }^{14}$ Ver http://www.dipres.gob.cl/597/articles-157545_doc_pdf.pdf.
} 
ciales y v) servicios de información. Estos productos involucran más de un programa presupuestario, como es el caso del producto sistema de financiamiento, que involucra los programas de Educación Superior y Gestión de Subvenciones de Establecimientos Educacionales, mientras que otros productos, como servicios asistenciales, sólo podrán asociarse al programa presupuestario Recursos Educativos, ${ }^{15}$ aun cuando podrían existir otros programas asociados, pero claramente no es posible la identificación de estos programas presupuestarios.

Caso contrario es el del Servicio Agrícola y Ganadero (SAG), que en su presupuesto del año 2017 posee siete programas presupuestarios, ${ }^{16}$ y la Corporación Nacional Forestal (Conaf), ${ }^{17}$ cinco programas presupuestarios, donde, en ambos casos, es posible vincular, de manera más clara, los recursos destinados en un programa presupuestario con los productos estratégicos, ${ }^{18}$ aunque aún existen espacios de mejora. También, en ambas instituciones, el respectivo programa 01 incorpora los recursos destinados principalmente a la administración de cada institución, los que no pueden ser vinculados directamente con los otros programas presupuestarios.

El análisis anterior muestra que es necesario homogeneizar la definición y operacionalización de los distintos programas presupuestarios incorporados en la Ley de Presupuestos, de modo que sea posible vincular más adecuadamente los recursos con los objetivos estratégicos y los productos que entrega cada institución. Esto implica, en algunos casos, abrir una mayor cantidad de programas en el presupuesto. Ésta es una propuesta de la Comisión, también de la OECD, tanto en el ya citado "Budgeting in Chile", ${ }^{19}$ como en el documento "Selected budget-

${ }^{15}$ Ley de Presupuestos 2017, partida 09, capítulo 01, programa 11.

${ }^{16}$ Ley de Presupuestos 2017, partida 13, capítulo 04, programa 01 (Servicio Agrícola Ganadero), programa 04 (Inspección de Exportaciones Silvoagropecuarias), programa 05 (Desarrollo Ganadero), programa 06 (Vigilancia y Control Silvoagrícola), programa 07 (Control Fronterizo), programa 08 (Gestión y Conservación de Recursos Naturales Renovables) y programa 09 (Laboratorios).

${ }^{17}$ Ley de Presupuestos 2017, partida 13, capítulo 05, programa 01 (Corporación Nacional Forestal), programa 03 (Manejo del Fuego), programa 04 (Áreas Silvestres Protegidas), programa 05 (Gestión Forestal) y programa 06 (Arborización Urbana).

18 Ver http://www.dipres.gob.cl/597/articles-157587_doc_pdf.pdf/ y http:// www.dipres.gob.cl/597/articles-157588_doc_pdf.pdf/.

19 Vammalle y Ruiz Rivadeneira, "Budgeting in Chile". 
ing issues in Chile: Performance budgeting, medium-term budgeting, budget flexibility". ${ }^{20}$

Es importante que la revisión de los productos estratégicos y la creación o eliminación de programas presupuestarios se realicen en el marco de la planificación estratégica y el sistema de planificación y control de gestión. Este proceso debe ser la base de la gradual transformación de la estructura presupuestaria y de la implementación de un correcto sistema de control de gestión, que utilice tanto la información de desempeño generada por la Dipres como aquélla puesta a disposición por el Ministerio de Desarrollo Social.

Junto con esto, es importante tener presente que el sistema de control de gestión debe permitir, además de vincular los productos y subproductos estratégicos de cada institución con los recursos destinados, la elaboración de indicadores de desempeño de distintos niveles de resultados (insumo, proceso, producto, resultado intermedio y resultado final); junto con la medición de la satisfacción con los productos, es decir, con los bienes y servicios recibidos. Se está de acuerdo con lo planteado por la Comisión respecto a que se debe elaborar una metodología común y transparente para la medición de satisfacción ciudadana, la que debe realizarse de manera sistemática y con una periodicidad establecida. Sin embargo, no es claro que todo deba agruparse en una plataforma única asociada a la División de Gobierno Digital y Tecnologías (134), dado que la información de satisfacción de los usuarios debe ser parte del sistema de control de gestión, y, por tanto, puede ser recogida por cada servicio público, pero bajo las pautas y supervisión de la División de Gobierno Digital y Tecnologías.

Adicionalmente, el sistema de control de gestión debe definir productos y subproductos estratégicos que se vinculen fácilmente con los programas del presupuesto, los que, a su vez, deberían corresponder a programas nuevos y reformulados que son evaluados como parte de la formulación presupuestaria (evaluación ex ante), y a los que se les realiza una evaluación más tarde ex post de sus resultados. La vinculación con el programa presupuestario puede ser el programa presupuestario completo o los ítems del subtítulo 24, el cual identifica las acciones

${ }^{20}$ Ian Hawkesworth, Óscar Huerta Melchor y Marc Robinson, "Selected budgeting issues in Chile: Performance budgeting, medium-term budgeting, budget flexibility", OECD Journal on Budgeting 12, n. 3 (2013). 
programáticas, según sea el nivel de agregación que se defina en el programa. A modo de ejemplo, el programa presupuestario 06 "Sistema de Protección Integral a la Infancia", de la Subsecretaría de Servicios Sociales del Ministerio de Desarrollo Social, corresponde a un producto estratégico. En su subtítulo 24 es posible identificar en cada ítem y asignación un conjunto de acciones programáticas (o subproductos), las que a su vez pueden ser evaluadas y contar con indicadores de desempeño.

Por tanto, el rediseño del sistema de control de gestión pasa por perfeccionar su vinculación con la estructura programática del presupuesto, pero haciendo los cambios en la unidad de análisis y usando adecuadamente la información que entrega el sistema de evaluación y control de gestión actualmente vigente en Chile. Éste se compone de: evaluaciones ex ante de programas sociales nuevos o reformulados y de proyectos de inversión pública, a cargo de la Subsecretaría de Evaluación Social del Ministerio de Desarrollo Social (MDS); la evaluación ex ante de los programas no sociales nuevos como también de ampliaciones y reformulaciones de programas existentes, realizadas por la Dipres; y la evaluación ex post de programas — que incluyen el diseño, gestión e impacto de los mismos - desarrollados por la misma Dipres, a través de evaluadores externos. Adicionalmente, se debe considerar que forman parte de este sistema los instrumentos de seguimiento o monitoreo del desempeño, en los que se incluyen la revisión del cumplimiento de metas gubernamentales por parte de la Segpres; el seguimiento de indicadores de desempeño con la revisión del cumplimiento de las metas respectivas, por parte de la Dipres; y el monitoreo de programas sociales realizado por la mencionada Subsecretaría de Evaluación Social del MDS.

\section{ESTABLECER LA AGENCIA DE PRODUCTIVIDAD Y EVALUACIÓN}

En lo que se refiere a la evaluación de resultados (intermedios y finales), hoy se cuenta con evaluaciones de impacto encargadas a evaluadores externos por la Dipres, en el marco de lo que normalmente se compromete en el Protocolo de Acuerdo con el Congreso y las evaluaciones de impacto que las propias instituciones licitan, a través de recursos considerados en el subtítulo 22, en la subasignación correspondiente a estudios.

La Comisión propone la creación de una Agencia de Productividad y Evaluación que goce de autonomía legal, para que se aboque a la 
evaluación ex post de políticas públicas, manteniendo en el poder ejecutivo el monitoreo permanente y la evaluación ex ante. Esta propuesta se explicaría dada "la dificultad del Ejecutivo de evaluarse a sí mismo con ecuanimidad (en particular cuando es frecuente que la Dipres se involucre profundamente en el diseño de los programas)" (121). Sin embargo, esta afirmación, en primer lugar, no toma en cuenta que las evaluaciones ex post de programas son mandatadas a través de licitaciones públicas, para efectuarse por instituciones académicas, centros de estudios y consultoras, con alta rigurosidad profesional. Por tanto, no se trata de una "autoevaluación del ejecutivo". En segundo lugar, asume que la Dipres participa en el diseño del programa, lo que no es así, pues la División de Control de Gestión de la Dipres o la División de Políticas Sociales de la Subsecretaría de Evaluación Social son las encargadas de evaluar los programas nuevos y reformulaciones planteados por los servicios, y no participan de su diseño.

Adicionalmente, se considera que para que los resultados de una evaluación ex post (incluyendo las evaluaciones de impacto) sean efectivos, éstos deben ser considerados en el proceso de toma de decisiones del rediseño o término de un programa. Este proceso es inherente a las responsabilidades del poder ejecutivo, por lo que, si se entiende que el fin último es "evaluar para mejorar", a juicio de esta autora la nueva institucionalidad de evaluación debe localizarse al interior del gobierno, pues debe tener la jerarquía y la posibilidad cierta de interactuar con las instituciones en el ciclo de definición de políticas y disponer de facultades para que las instituciones y programas evaluados se pronuncien respecto de las recomendaciones que entreguen las evaluaciones. La experiencia internacional muestra que los sistemas de evaluación exitosos están a cargo de una institución con liderazgo y alta capacidad técnica, validada por toda la administración pública. Los liderazgos que sobresalen han sido aquéllos situados en los círculos del centro de gobierno y del proceso presupuestario, lo que en muchos países es un rol de los ministerios de finanzas o similares. ${ }^{21}$ Así, en casos como los de Canadá, Estados Unidos y el Reino Unido, entre otros, existe una función de evaluación incorporada en la institucionalidad a cargo de las

${ }^{21}$ Ver Keith Mackay, "Institutionalization of Monitoring and Evaluation Systems to Improve Public Sector Management”, Evaluation Capacity Development Working Paper Series n. ${ }^{\circ}$ 15, World Bank (Washington DC, 2006). 
finanzas públicas. En Canadá se trata del Treasury Board Secretariat; en Estados Unidos, de la Office of Management and Budget (OMB), y en el Reino Unido, el Treasury Board. Por tanto, lo que se propone es que exista un servicio público a cargo de las evaluaciones, que se relacione con el Ministerio de Hacienda, y posea la misma jerarquía institucional que hoy tiene la Dipres.

En relación con la propuesta de la Comisión, según la cual la Agencia debería elaborar anualmente "un plan de evaluación, de manera que su agenda esté programada con antelación y sea de conocimiento público", y que dicho plan "sea fijado entre el Congreso, el Ejecutivo y la propia Agencia" (123), se considera que lo planteado ocurre hoy, al establecer los programas que anualmente serán evaluados de manera ex post como parte de la discusión del proyecto de Ley de Presupuestos de cada año, generalmente en el Protocolo de Acuerdo. Adicionalmente, la información que surge de dichas evaluaciones es pública y presentada de manera sistematizada a través de resúmenes ejecutivos que acompañan al proyecto de Ley de Presupuestos, y, por tanto, permite al Congreso ejercer su rol fiscalizador.

\section{AUMENTAR LA TRANSPARENCIA Y MEJORAR LA CALIDAD DE LA INFORMACIÓN DE EVALUACIÓN DE DESEMPEÑO}

La Comisión señala que la información que "actualmente publica la Dipres no es suficientemente detallada ni tampoco está adecuadamente presentada de modo de facilitar el uso de la información difundida" (130). Este problema, en parte, se debe a lo analizado en esta reseña respecto a la desvinculación que existe entre la información de desempeño y la estructura presupuestaria. Por tanto, con las propuestas analizadas y las sugerencias adicionales de esta autora en relación a la planificación estratégica y al rediseño del sistema de control de gestión y la estructura presupuestaria, este aspecto del problema estaría resuelto.

Sin embargo, se debe avanzar en que tanto la información de gasto como de desempeño sea presentada en formato de base de datos, tal que permita elaborar series de tiempo de indicadores claves, como costo unitario de los bienes y servicios provistos, o porcentaje de gastos administrativos. 
Además, la Comisión propone que la ejecución del gasto público sea presentada con la mayor desagregación posible y en formatos que permitan al usuario agrupar y resumir información de acuerdo a sus propósitos (131). Estando de acuerdo con esta propuesta, se debe revisar que el nivel de desagregación no atente contra la adecuada vinculación de cada programa presupuestario con el producto estratégico o los subproductos a los cuales se les definirá un indicador de desempeño.

\section{VELAR POR LA USABILIDAD DE LOS DATOS QUE SE TRANSPARENTAN}

Se está de acuerdo con la propuesta de la Comisión en relación con que "la publicación de los datos cuente con estándares mínimos de usabilidad y consistencia, siendo imprescindible presentar la información acumulada en el tiempo (series largas) y con la máxima desagregación territorial posible, si corresponde" (134).

Un avance en la usabilidad, sin duda, será contar con la vinculación de los programas presupuestarios con los productos estratégicos, tal como fue analizado en la sección de rediseño del sistema de control de gestión.

Adicionalmente, se considera que la información debería poder ser agrupada en distintas categorías. A la actual clasificación por tipo de gasto (como los habituales subtítulos de cada programa: personal, bienes y servicios, prestaciones de seguridad social, transferencias corrientes, adquisición de activos financieros, iniciativas de inversión, etcétera), se deberían sumar agrupaciones conforme a otras categorías, como la población a la que se dirige el gasto — por ejemplo, infancia, jóvenes, adultos mayores, mujeres, entre otros-; los objetivos estratégicos que se persiguen; el tipo de bien o servicio que se entrega - transferencia monetaria, asistencia técnica, etcétera-, o por área geográfica, en la que se ejecuta el gasto (región o comuna).

Este tipo de agrupaciones es actualmente utilizado en la plataforma del Banco Integrado de Programas Sociales ${ }^{22}$ en la que a partir del proceso de seguimiento es posible agrupar programas por ámbito de acción, condiciones de beneficiarios, tipo de beneficios y ciclo de vida.

22 Ver http://www.programassociales.cl/programas/. 


\section{CREAR INCENTIVOS ALAHORRO EN LA GESTIÓN PRESUPUESTARIA}

La Comisión plantea que actualmente "existe un claro desincentivo a los jefes de servicio a buscar espacios para ahorros y eventuales reasignaciones internas" (82). Sin embargo, esta premisa no considera que los jefes de servicios normalmente realizan reasignaciones durante el año, en acuerdo con la Dipres, para una más eficiente y adecuada ejecución del gasto.

Dado este supuesto desincentivo, la Comisión propone implementar "un sistema de ahorros compartidos a partir de ganancias de eficiencia en la gestión de compromisos de gasto permanente. En un año determinado, una vez cumplidas las metas de servicios o productos definidas en el presupuesto, el 50 por ciento de los eventuales excedentes en los subtítulos de gasto permanente del programa podrían ser parcialmente enterados al presupuesto del servicio del año subsiguiente" (83).

Esta autora no está de acuerdo con lo planteado por la Comisión, principalmente por cuatro razones. La primera: los gastos permanentes se encuentran asociados a leyes permanentes, que obligan a la entrega de un cierto bien o servicio a todos aquellos usuarios o beneficiarios que cumplan con los requisitos de elegibilidad (por ejemplo, para subvención escolar, pensión básica solidaria, entre otros). Estos recursos están normalmente identificados en el subtítulo 24 , y su monto viene dado por la ley permanente que los creó. Por tanto, la generación de ahorro sólo podría venir por disminución de cobertura, lo que perjudicaría a las personas.

La segunda: el comprometer recursos entre un periodo presupuestario y otro para un uso que no está claramente identificado en la Ley de Presupuestos desconoce que el proceso de discusión del Proyecto de Ley de Presupuestos significa el priorizar el uso de recursos, y establecerlo en una ley, con aprobación del parlamento.

La tercera: puesto que los ahorros de gasto obtenidos podrían ocuparse en el presupuesto de años posteriores, se podría contravenir la regla fiscal y, por tanto, la promesa de entrega de esos ahorros no ser factible de implementarse. Así lo señala Steger al analizar el caso austríaco, ${ }^{23}$ en el que ajustes fiscales, debido a las condiciones macro-

${ }^{23}$ Gerhard Steger, "Elaboración de presupuesto por resultados: lecciones de la reforma presupuestaria austriaca", en Un Estado para la ciudadanía. Estudios para su modernización, editado por Isabel Aninat y Slaven Razmilic (Santiago: Centro de Estudios Públicos, 2018), 489-532. 
económicas, no permitieron entregar las reservas generadas por los ahorros.

Por último, la cuarta razón se refiere al incentivo perverso que pueden tener los servicios al comprometer, en términos de desempeño, menos de lo que es posible alcanzar con los recursos asignados a un cierto programa, de modo de cumplir la meta y obtener "ahorros". Este último caso podría evitarse con la existencia del sistema de control de gestión que se discute en detalle en esta reseña.

Con todo, si se desea analizar estrategias para incentivar el ahorro, las mismas no deberían basarse en el gasto permanente, sino que en el gasto operacional, especialmente el identificado en el subtítulo 22 .

\section{GENERAR MÁS ESPACIOS DE FLEXIBILIDAD EN LA EJECUCIÓN DEL PRESUPUESTO}

Al analizar posibles formas de aumentar la flexibilidad en la ejecución presupuestaria de los servicios públicos es necesario partir de la base de que, en términos comparados con los países de la OECD, Chile posee una adecuada flexibilidad. ${ }^{24} \mathrm{Si}$ bien las reasignaciones deben ser aprobadas por la Dipres, lo cierto es que en la mayoría de los casos las propuestas surgen desde los propios servicios.

A pesar de lo anterior, la Comisión propone dos grandes medidas para aumentar la flexibilidad: i) reducir la desagregación del subtítulo 29 (adquisición de activos no financieros), dejándolo sólo a nivel de subtítulo, y ii) disminuir el número de asignaciones en los subtítulos 24 (transferencias corrientes) y 33 (transferencias de capital), apuntando a que tengan un menor nivel de detalle.

Respecto de la primera propuesta de la Comisión, no se está de acuerdo con reducir la desagregación del subtítulo 29, pues el gasto en tecnología (hardware y software) es cada vez más importante para la correcta entrega de los bienes y servicios, requiriéndose continuamente el desarrollo de diversas plataformas. Este desarrollo debe ser evaluado, de manera que no existan duplicaciones; y tal como lo plantea la Comisión, en esta evaluación debería participar a lo menos la Unidad de Modernización de la Segpres y la Dipres, y avanzar a que exista una Dirección de Gobierno Digital y Tecnologías de la Información. Por tanto,

${ }^{24}$ Ver Vammalle y Ruiz Rivadeneira, "Budgeting in Chile". 
la flexibilización del subtítulo 29 atentaría contra la correcta ejecución de las iniciativas a identificar en este subtítulo, que deberían ser sólo aquéllas que hayan sido debidamente evaluadas.

Respecto de la recomendación de agregar los subtítulos 24 (transferencias corrientes) y 33 (transferencias de capital), ella es inconsistente con lo planteado en relación con acceder a información con la mayor desagregación posible (en sección de transparencia y usabilidad de la información). Al mismo tiempo, el nivel de apertura en asignaciones de los subtítulos 24 y 33 debe evaluarse tomando en consideración la necesidad de reestructurar los programas presupuestarios para vincularlos con los productos y subproductos estratégicos. Así, en la mayoría de los casos, un programa presupuestario debería corresponder a un producto estratégico (por ejemplo, financiamiento de la educación superior), y los subproductos deberían identificarse a nivel de asignación al interior del subtítulo (por ejemplo: gratuidad, becas, crédito, entre otros).

Por otra parte, a la hora de evaluar la entrega de mayores niveles de flexibilidad en la ejecución del presupuesto de los servicios, es importante poner como primer requisito el cumplimiento de los resultados que se hayan definido en el sistema de control de gestión. En ese sentido, se debería revisar en detalle la experiencia, que existió entre 2004 y 2008, de entrega de mayor flexibilidad como premio a aquellos servicios que, junto con cumplir con el 100 por ciento del Programa de Mejoramiento de Gestión (PMG), cumplieron con la disciplina financiera, respetando, entre otras cosas, la programación de caja. En esos años la flexibilidad presupuestaria venía dada por: i) traspaso de recursos presupuestarios - originados en mayores ingresos o ahorros permanentes - de un año fiscal al siguiente, en la forma de un saldo inicial de caja por sobre la estimación del presupuesto aprobado, y ii) traspaso de recursos entre proyectos de inversión; es decir, flexibilidad para reasignar recursos de los ítems 61 al 74 del subtítulo 31 (antes denominado como inversión real, ahora como iniciativas de inversión), en la medida en que mantengan la recomendación técnico-económica favorable (RS) del Mideplan, actualmente Ministerio de Desarrollo Social, y hasta por el monto autorizado en la Ley de Presupuestos. ${ }^{25}$ Durante este periodo,

${ }^{25}$ Dirección de Presupuestos, Informe de Finanzas Públicas. Proyecto de Ley de Presupuestos del Sector Público para el año 2006 (Santiago: Ministerio de Hacienda, Gobierno de Chile, 2005), 151. 
existieron servicios que utilizaron la flexibilidad tipo i. En el caso de la flexibilidad ii, ella fue utilizada independientemente del cumplimiento del PMG, dado que la reasignación de los proyectos de inversión permite una eficiente ejecución del presupuesto, dadas las contingencias que pueden ocurrir en la implementación de las iniciativas de inversión.

La modernización del Estado debe entenderse como un proceso de mejora continua $y$, en ese sentido, esta reseña ha buscado hacer un análisis de las propuestas de la Comisión considerando lo avanzado y los desafíos que debemos abordar para hacer más eficiente, eficaz y con mayor calidad la entrega de bienes y servicios a la ciudadanía. Al mismo tiempo, propone herramientas que permitan el adecuado seguimiento del cumplimiento de los objetivos propuestos, tanto desde un punto de vista financiero como de sus resultados.

\section{BIBLIOGRAFÍA}

Aninat, Isabel y Slaven Razmilic, coordinadores. Un Estado para la ciudadanía. Informe de la Comisión de Modernización del Estado. Santiago: Centro de Estudios Públicos, 2017.

, editores. Un Estado para la ciudadanía. Estudios para su modernización. Santiago: Centro de Estudios Públicos, 2018.

Arenas, Alberto \& Heidi Berner. Presupuesto por resultados y la consolidación del Sistema de Evaluación y Control de Gestión del gobierno central. Santiago: Dirección de Presupuestos, Ministerio de Hacienda de Chile, 2010.

Dirección de Presupuestos. Informe de Finanzas Públicas. Proyecto de Ley de Presupuestos del Sector Público para el año 2006. Santiago: Ministerio de Hacienda, 2005. http://www.dipres.gob.cl/598/articles-37447_doc_pdf.pdf/.

Hawkesworth, Ian, Óscar Huerta Melchor \& Marc Robinson. "Selected budgeting issues in Chile: Performance budgeting, medium-term budgeting, budget flexibility". OECD Journal on Budgeting 12, n. 3 (2013).

Mackay, Keith. "Institutionalization of Monitoring and Evaluation Systems to Improve Public Sector Management". Evaluation Capacity Development Working Paper Series n. ${ }^{\circ}$ 15. World Bank (Washington DC, 2006).

Steger, Gerhard. "Elaboración de presupuesto por resultados: lecciones de la reforma presupuestaria austriaca". En Un Estado para la ciudadanía, editado por Aninat \& Razmilic.

Vammalle, Camila \& Ana María Ruiz Rivadeneira. "Budgeting in Chile". OECD Journal on Budgeting 2016, n. ${ }^{\circ} 3$ (2017).

Von Gersdorff, Hermann. "El proceso presupuestario de Chile: opciones de reforma". En Un Estado para la ciudadanía, editado por Aninat \& Razmilic. EP 